\title{
Scripta
}

\section{Bari e gli studi catalani}

\author{
Bari and Catalan studies
}

AnNAMARIa ANNicCHIARICO

annamaria.annicchiarico@uniroma3.it

Università Roma Tre

Riassunto: Il contributo ricostruisce la tradizione degli studi catalani che l'Università di Bari, e segnatamente la cattedra di Filologia Romanza coperta da Giuseppe Edoardo Sansone, ha visto nascere tra fine anni '50 e inizi anni '60, e poi fiorire nei decenni successivi. Aprendo alla catalanistica le porte del mondo accademico molto precocemente, si creava a Bari non solo uno spazio accademico di ricerca, ma anche un luogo di promozione e di risonanza nazionale e internazionale per la catalanistica. Si ricostruiscono, a grandi tratti, i principali filoni di ricerca che hanno visto l'impegno del gruppo barese primigenio, ricordando i contributi fondamentali dei singoli componenti e le principali linee di ricerca che ciascuno di essi ha sviluppato in prosieguo di tempo.

Parole chiave: Filologia Romanza, lingua e letteratura catalana, Università di Bari

Abstract: This contribution aims at tracing the tradition of Catalan studies that the University of Bari, with the chair of Romance Philology covered by Giuseppe E. Sansone, saw being born in the late 1950s - early 1960s at, and then flourished in the following decades. By opening the doors of the academic world to Catalanism very early, Bari was not only creating an academic space for research, but also a place of promotion and national and international resonance for Catalanism. The main lines of research faced with by the original Bari research group are recalled, together with the fundamental contributions of the individual components and the main lines of research that each of them has developed over time.

Keywords: Romance Philology, catalan language and literature, University of Bari 
Mi sembra che la felice circostanza di un Convegno a Bari di catalanistica sia un'occasione da non perdere per guardare indietro negli anni e ricostruire la tradizione, appunto, degli studi catalani che questa sede universitaria ha visto nascere e fiorire. Tanto più che la prima giornata del Convegno prevede interventi concernenti i secoli fondativi della civiltà letteraria catalana (1100-1400) e, segnatamente, autori ed opere che, ben mezzo secolo fa, erano già all'attenzione di studiosi che qui hanno trascorso tutta la loro vita accademica, o parte di essa.

Me compresa, che sono d'origine in parte barese; e qui, a Bari, ho studiato e iniziato la carriera accademica, quasi da subito orientata verso interessi, appunto, catalanistici.

Colgo l'occasione, dunque, per una breve ricognizione del passato «locale»; e lo farò ben volentieri, partendo dal dato di fatto che questa sede può vantarsi di aver aperto alla catalanistica le porte del mondo accademico molto precocemente.

Gli inizi degli studi sulla civiltà letteraria catalana a Bari, infatti, ci portano molto indietro nel tempo; diciamo verso la fine degli anni '40. Con un punto di partenza, ideale, per Bari, che non è propriamente Bari, ma Firenze.

È a Firenze infatti; dove negli anni '48-'49, il giovane studioso, Giuseppe Edoardo Sansone, sta seguendo dei corsi di specializzazione in Filologia Romanza. Qui incontra Mario Casella, e qui, probabilmente tramite lo stesso Casella, che già dalla seconda decade del ' 900 aveva prodotto scritti d'interesse catalanistico, prende i contatti con la Catalogna. ${ }^{1}$

Ed è da qui infatti, proprio da Firenze, che il giovane parte, per divenire, nel giro di pochi anni, curiosissimo cacciatore di carte, manoscritti, incunaboli e antiche edizioni della Biblioteca de Catalunya. E non a caso, avrebbe intitolato «Fra carte e manoscritti nella Biblioteca de Catalunya» la prima sezione del volume Studi di Filologia Catalana del 1963, nel quale raccoglieva saggi redatti tra il 1955-1956 e il 1962. Tant'è che, tra la frequentazione di questa Biblioteca e i contatti con l'Institut d'Estudis Catalans, instaurava relazioni di amicizia con quelli studiosi catalani che per lui saranno gli amici di una vita; e, per i suoi allievi a venire, noi, punti di riferimento costante: da Ramon Aramon i Serra, a Pere Bohigas, a Josep Maria de Casacuberta, a Martí de Riquer. Nel frattempo, il XII premio internazionale «Enric de Larratea» conferitogli dall'Institut d'Estudis Catalans (Secció Filologica), nel 1963, per la summenzionata raccolta di saggi, rappresentava un riconoscimento importante non solo all'attività dello studioso, ma anche all'operosità del divulgatore e maestro di studi catalani. $\mathrm{Al}$ contempo, indirettamente, poneva all'attenzione internazionale, insieme allo studioso, la cattedra

1 Per una ricostruzione esaustiva del profilo di G. E. Sansone come filologo romanzo, rinvio a C. Segre, «Giuseppe Edoardo Sansone», in Parola del Testo, Anno VIII, 2004/1. Studi in onore di Giuseppe Sansone, vol. I, pp.7-10; C. Zilli, «Giuseppe E. Sansone (1925-2003)», in Estudis Romànics, XXVII, 2005, pp.596-602. Allo stesso modo, per il profilo di catalanista di Mario Casella, qui più volte citato, rinvio a: O. Macrì, «Mario Casella, ispanista», in Studi Danteschi, LIX, 1987, pp. 93-169; L. Formisano, «Casella studioso di letteratura catalana», in Studi Danteschi, 59, 1987, pp.57-65; P. Gómez Soler, «Il rapporto di Mario Casella con la cultura catalana», in Bollettino Storico Piacentino, Anno CVII, 2, 2012, pp.290-317. 
(l'«Istituto») di Filologia Romanza, di questa università, come spazio accademico di ricerca e di promozione per la catalanistica medievale e moderna.

A questo punto, il dado era tratto. Con Sansone chiamato come professore incaricato nel 1952, e poi come ordinario di Filologia Romanza nel 1960, il catalano de facto arrivava a Bari, sia nella attività scientifica della scuola, sia nell'attività didattica in senso lato (programmi d'esami all'interno della disciplina, tesi di laurea, seminari). Negli stessi termini, successivamente, conduttore sempre Sansone e sotto l'egida della filologia romanza, «il catalano di Bari», diciamo così, approdava anche all'Università di Pescara (1969), poi alla Facoltà di Lettere dell'Istituto Universitario Orientale di Napoli nel 1971, poi nel 1974 alla Facoltà di Magistero di Roma (divenuta in seguito Facoltà di Lettere e Filosofia dell'Università Roma Tre).

Bari, dunque, riprendo il filo della mia ricognizione, vede la nascita, tra fine '50 e inizi ' 60 , di quel gruppo di ricerca, che poi sarebbe rimasto attivo nei decenni successivi. Come dire nasce una officina barese, che ben presto si mostrerà impegnata su un ampio ventaglio di filoni di ricerca, spalmato prevalentemente, ma non soltanto, sui secoli di mezzo.

Ecco ora una rapida occhiata ai primi prodotti e, quindi, alle linee di ricerca baresi. A cominciare dal succitato volume del Sansone pubblicato nella collana «La Biblioteca di Filologia Romanza», da lui stesso fondata presso l'Adriatica Editrice di Bari. Collana che poi avrebbe ospitato altri volumi, tra edizioni e raccolte di saggi, di catalanistica. A riguardo, osservo che, proprio nel volume in discorso, figura un saggio, precedentemente pubblicato in Revista de Filología Española (1960), dedicato ad uno degli autori, che è all'attenzione di questo Convegno: Ramon Llull; «Ramon Llull narratore», ne era il titolo, infatti. Come pure, nell'ottica della ricostruzione storica assunta da questo intervento, è altrettanto significativa la pubblicazione, a cura dello stesso Sansone, dei Saggi di letteratura provenzale e catalana di Casella, appunto, nel 1966, nella stessa collana.

Parlavo della officina barese e del quadro articolato dei filoni d'interesse e di ricerca in essa coltivati. Certo. A iniziare dalla letteratura medievale catalana scientifica, o pseudoscientifica. Si tratta di un settore ampiamente frequentato. Soprattutto da Vincenzo Minervini. Ne ricordo alcuni saggi, di fine anni ottanta / inizi novanta: «Les virtuts de l'aygua ardent» (1989); «Il Flos de cosmetica catalana» (1991); gli "Appunti sui Començaments de medicina lulliani» (1992). Così come, per la letteratura farmacologico-dietologica, ricordo l'edizione di Maria Luisa Indini: «Un Tractat de les viandes e dels beures nella dietologia medievale catalana» (1991).

Rappresentato, a Bari, è stato anche il settore, contiguo al precedente, dell'enciclopedismo catalano. E ricordo, a riguardo, l'edizione della versione catalana del Libro di Sidrac, pubblicata da Minervini nel 1982 (cui seguirà nel 2010 l'edizione, sempre a cura di Minervini, di una redazione del medesimo testo in lingua d'oc: $I l$ «Libre de Sidra@ 'di' Bertran Boysset). Contestualmente, per la trattatistica sempre di base enciclopedica, ma con carattere allegorico-edificante, ricordo Il «Livre des Bestes» di Ramon Llull, versione francese dell'omonimo testo catalano, pubblicata dal Sansone nel 
1964. Nonché l'edizione in due volumi dei Bestiaris, curata da Saverio Panunzio (1963-1964). Due volumi che sono, piace sottolinearlo, due volumetti, ancora in dodicesimo all'epoca, della collana Els Nostres Classics; della Editorial Barcino; quella che per tutti era semplicemente, affettivamente, «la collana di Casacuberta». Fondata nel 1924, durante la dittatura di Primo de Rivera, dal Casacuberta appunto, e con quella intitolazione in cui il possessivo - els nostres - denotava l'orgoglioso senso di attaccamento al proprio patrimonio culturale e identitario. Nello stesso torno di anni, e ancora nella stessa veste editoriale, Sansone pubblicava l'edizione in due volumi del Cercapou (1957-1958), e l'edizione della versione catalana del Liber consolationis et consilii di Albertano da Brescia (1965).

Anche il filone dei volgarizzamenti e delle traduzioni è stato frequentato, dagli anni ' 60 in poi, dal gruppo barese, come attestano studi e edizioni critiche varie. Va da sé che parliamo di traduzioni nel senso medievale; ovverosia di traduzioni per quali «non c'è da stupirsi per il fatto che il lieve diaframma che nell'Età di mezzo separa traduzione (o volgarizzamento) e rifacimento sia con frequenza infranto: al contrario si potrebbe dire che la versione medievale è istituzionalmente un rifacimento» (A. D'Agostino, «Traduzione e rifacimento», in AA.VV., Testo medievale e traduzione, Bergamo University Press-Edizioni Sestante 2001, p.155). Ricordo, in merito a questo ambito di ricerca, e in aggiunta a qualche titolo già menzionato, l'edizione del Livre de l'ordre de chevalerie, versione francese del testo lulliano; quindi, l'edizione della versione catalana della Cárcel de Amor di Diego de San Pedro; quindi ancora l'edizione del Llibre del plant de l'hom, versione catalana del Liber de miseria humanae conditionis di Lotario Diacono. Edizioni tutte e tre, queste ultime, a cura di V. Minervini, rispettivamente nel 1972 (Adriatica Editrice), 1986 (Schena Editore), 1996 (Schena Editore). E ancora la mia edizione della Fiammetta catalana (Japadre Editore, 1983-1987). Edizione che poi mi ha portata, in prosieguo di tempo, a studiare la presenza del Boccaccio, e segnatamente della Fiammetta, nella narrativa (dal Tirant a Roís de Corella) e nello specifico della cosiddetta novela o ficció sentimental (con l'edizione del Frondino e Brisona, Adriatica Editrice, 1990).

A questo punto, molto altro si potrebbe dire circa la pluralità degli indirizzi di ricerca precocemente inaugurati tra gli anni 50-60 dal Sansone a Bari, dentro il medioevo, e fuori dal medioevo con la poesia del ' $9 \mathrm{OO}$, con speciale attenzione a Carles Riba, e con le avanguardie storiche; indirizzi che continuarono ad essere coltivati negli anni successivi. A riguardo basti ricordare, fra l'altro, i suoi saggi e interventi sulla traduzione poetica e le varie antologie con traduzione a fronte (Poesia catalana del 900, Newton- Compton, 1979; «Poesia catalana», in AA. VV. Parnaso Europeo, Lucarini, II, 1989 e II, 1990; le sezioni curate in Antologia de poetes catalans, Galàxia Gutemberg, III e IV, 1997; Poesia catalana del Medioevo, Interlina, 2001)

Detto ciò, in questa sede, mi sembra più pertinente, e coerente con il programma del Convegno, ricostruire il contributo dato dal gruppo barese agli studi riguardanti gli autori, o le opere, o i fenomeni, di cui si parlerà nel Convegno. Ovverosia, Ausiàs March, in lui compresa l'eredità trobadorica e post-trobadorica, e il Tirant lo Blanch.

A iniziare dai saggi di Panunzio degli anni ' 80 dedicati ad Ausiàs March e al riuso marquiano della 
tradizione didattico-allegorica dei bestiari medievali («Una cançó emblemàtica d'Ausiàs March», 1980; «Realisme i didactisme en les comparacions d'Ausiàs March pròpies del Bestiari», 1983). A finire con quelli di Sansone. Per quest'ultimo, ricordo gli studi sulla struttura metrico-ritmica del canzoniere, e saggi vari, intesi a interpretare singoli canti, o gruppi di canti. Quindi, ancora, i lavori del suddetto sulla ricezione di March; ovverosia sulle versioni cinquecentesche, da Romaní all'Anonimo. Va da sé che Ausiàs March entri indirettamente da varie porte, e in ogni dove: come negli studi centrati sulla narrativa breve e lunga, in specie quelli sulla cosiddetta ficció sentimental; così nei miei contributi sulle «intertestualità» fra Corella, Ausiàs March, Tirant, Fiammetta: un seducente ménage a due, a tre, a quattro, generalmente su base ovidiana.

Ed eccoci al Tirant. Al centro degli interessi di Sansone dal 1960 (anno di pubblicazione del saggio sul notissimo giudizio cervantino, poi in Saggi iberici, Adriatica Editrice, 1974), il Tirant ha continuato ad essere a lungo indagato dallo studioso. In saggi che hanno riguardato l'analisi dei modi e delle forme del narrare di Martorell, o che hanno affrontato i momenti più di spicco della fortuna postuma del romanzo. Mi riferisco, circa quest'ultima, all'importante traduzione cinquecentesca di Lelio Manfredi e a quella, non meno rilevante, settecentesca del Comte di Caylus. A quest'ultimo filone d'indagine sono riconducibili anche i saggi di Carmelo Zilli raccolti nel volume Manfrediana (Adriatica Editrice, 1991). A questo punto, senza dilungarmi sui contributi dei singoli studiosi al Tirant/Tirante; non mi resta che terminare questo excursus, il cui obiettivo è quello di ricostruire, a grandi tratti come dicevo, i primordi degli studi catalani a Bari e le principali linee di ricerca attorno alle quali essi si sono sviluppati. Chiudo, pertanto, con quella che resta la testimonianza più tangibile, diciamo, della dedizione alla catalanistica, di tutti i componenti insieme della pattuglia barese primigenia (A. Annicchiarico, M.L. Indini; M. Majorano, S. Panunzio, V. Minervini, C. Zilli): ovverosia, l' edizione della versione italiana del Tirant lo Blanch di Lelio Manfredi, pubblicata con Introduæione di G. E. Sansone nel 1984 (Tirante il bianco. Romanzo cavalleresco del XVI secolo, La Tipografica, Roma).

Al contempo, formulo i migliori auspici per la nuova stagione di studi catalani che si è instaurata da alcuni anni a Bari, e che, in sinergia con l'ispanistica e la filologia romanza, sarà sicuramente ricca di prospettive di ricerca e feconda di risultati. 